\title{
Dynamics of Type-II Supernovae
}

\author{
By H. - Th. J A N K AND E. M. MÜLLER
}

Max-Planck-Institut für Astrophysik, Karl-Schwarzshild-Strasse 1, D-8046 Garching, Germany

Hydrodynamical simulations of type-II supernovae in one and two dimensions are performed for the revival phase of the delayed shock by neutrino energy deposition. Starting with a postcollapse model of the $1.31 M_{\odot}$ iron core of a $15 M_{\odot}$ star immediately after the stagnation of the prompt shock about $10 \mathrm{~ms}$ after core bounce, the models are followed for several hundred milliseconds with varied neutrino fluxes from the neutrino sphere. The variation of the neutrino luminosities is motivated by the considerable increase of the neutrino emission due to convective processes inside and close to the neutrino sphere (see Janka 1993), which are driven by negative gradients of entropy and electron concentration left behind by the prompt shock (Burrows \& Fryxell 1992, Janka \& Müller 1992). The size of this luminosity increase remains to be quantitatively analyzed yet and may require multi-dimensional neutrino transport. However, in the presented simulations the region below the neutrino sphere is cut out and replaced by an inner boundary condition, so that the convective zone is only partially included and the neutrino flows are treated as a freely changeable energy source.

For small neutrino luminosities the energy transfer to the matter is insufficient to revive the stalled shock. However, there is a sharp transition to successful explosions, when the neutrino luminosities lie above some 'threshold value'. Once the shock is driven out and the density and temperature of the matter between neutrino sphere and shock start to decrease during the expansion, suitable conditions for further neutrino energy deposition are maintained, and an explosion results. With the neutrino energy deposition the entropy per nucleon in the region between neutrino sphere and shock grows, and convective overturn will set in. Multi-dimensional simulations show that due to the large pressure scale height a large-scale pattern of up-flows and down-flows with velocities close to the local speed of sound develops. Consequently, cold, postshock material is advected down into the neutrino heating layer close to the neutrino sphere and hot material is transported outwards, thus reducing energy losses by re-emission of neutrinos and increasing the pressure behind the shock. Therefore these convective processes are found to be a very important aid to the delayed supernova explosion. In fact, two-dimensional models explode even in cases where spherically symmetrical computations fail.

\section{Type-II Supernova Explosions and Convection}

According to the current understanding type-II supernova explosions can be driven by neutrino energy deposition in the layers between the nascent neutron star and the stalled prompt supernova shock (Bethe \& Wilson 1985, Wilson et al. 1986). The 'delayed' explosion is triggered on the neutrino heating time scale instead of the hydrodynamical time scale, i.e. within (several) 100 milliseconds rather than ten milliseconds after core bounce. Although the neutrinos streaming out of the neutrino decoupling region in the surface of the forming neutron star loose only a small fraction of their energy (at most a few per cent) in the surrounding stellar matter, this energy may be sufficient to 'revive' the core bounce shock, which transformed to a standing accretion shock when energy losses due to dissociation of iron nuclei and emission of neutrinos reduced the pressure in the post shock material. The principle viability of this mechanism seems to be proven. However, there are still crucial questions to be answered. Firstly, it is not well understood, how, and when, the shock revival occurs and what the possibility of a successful explosion is defined by. Controversial results by independent elaborate supernova simulations (Mayle 1985, Wilson et al. 1986; Bruenn 1989a,b, 1992) give a 
hint that an increase of the neutrino emission by convective processes inside the neutron star may be crucial: While Bruenn (1992) does not regard convection and does not get explosions, Mayle (1985) and Wilson et al. (1986) obtain explosions when they include mixing processes by neutron finger instabilities in a parametrical description in their one-dimensional simulations. Secondly, although neutrino heating is able to eject the envelope of the star, the explosion energies in the delayed scenario are too low compared with typical type-II energies as deduced from light curve analyses (see Wilson et al. 1986; Janka 1993). This defines a quantitative problem, which still remains to be settled.

Janka (1993) performed long-time hydrodynamical simulations of the post bounce supernova evolution until more than 10 seconds after the revival of the stalled shock. Neutrino heating external to the nascent neutron star was carefully taken into account and the neutrino emission from the neutron star was varied within 'reasonable' limits. The simulations showed that high explosion energies cannot be obtained through energy deposition by the slowly decaying neutrino emission during the Kelvin-Helmholtz cooling of the protoneutron star. Instead, it was found that energetic explosions require the occurrence of an early phase of high neutrino luminosities with efficient heating in the vicinity of the neutron star. This means that the answer to the energy problem has to be searched for in the first few hundred milliseconds after core bounce. In this sense neutrino-driven supernovae are exploded by a delayed mechanism, not a late one.

When the prompt shock propagates through the outer layers of the collapsed stellar iron core and a luminous outburst radiates away several $10^{56}$ electron neutrinos within about 10 milliseconds, negative gradients of entropy and lepton number originate, and can give rise to convective instabilities (Burrows 1987; Burrows \& Lattimer 1988). Twodimensional (Burrows \& Fryxell 1992, Janka \& Müller 1992) and three-dimensional simulations (Müller 1993) indeed show that these instabilities grow on a time scale of about 10 milliseconds and mix a region encompassing the neutrino sphere with maximum velocities close to the local speed of sound. After 10-20 milliseconds a region of about $0.5 M_{\odot}$ between 20 and 120 kilometers is involved in the convective overturn and is homogenized within about 20 milliseconds (see Janka \& Müller 1992). Neutrino decoupling occurs at densities $\rho \lesssim 10^{11} \mathrm{~g} / \mathrm{cm}^{3}$, which is inside the convective layer. Therefore a considerable increase of the neutrino emission must be associated with the transport of material with high electron concentration from regions at $\rho \gtrsim 10^{12} \mathrm{~g} / \mathrm{cm}^{3}$ into the neutrino-transparent regime. This convection takes place inside the forming neutron star and is not in direct contact with the exploding layers of the star. Of course, crucially determining the neutrino luminosities during the early post bounce phase, this convection is of immediate importance for the explosion of type-II supernovae in the context of the neutrino-heating mechanism.

Observations of ${ }^{56} \mathrm{Ni}$ mixed into the hydrogen-rich envelope in SN 1987A suggest that convective processes might have occurred also outside the protoneutron star at a very early stage during the supernova explosion. This could have brought material originally located close to the new-born neutron star with high velocities into the outer layers of the star. Herant et al. (1992) have shown that the energy deposition by neutrinos in the vicinity of the neutron star is able to drive large-scale convective overturn. Together with the turbulence phenomena inside the forming neutron star these instabilities introduce a new level of complexity in our picture of type-II supernova explosions.

The work presented here concentrates on the post-bounce history of the supernova shock. The behaviour of the stalled prompt shock wave under the influence of varied neutrino emission from the protoneutron star is studied by hydrodynamical simulations. One- and two-dimensional simulations are compared to gain evidence about the occur- 
rence and importance of turbulence outside the new-born neutron star. In Sect. 2 the model, physical assumptions and some numerical aspects are described, in Sect. 3 the results are discussed, and Sect. 4 ends with a summary and conclusions.

\section{Model, Physical Assumptions, and Numerical Aspects}

The post bounce evolution of the collapsed $1.31 M_{\odot}$ iron core of a $15 M_{\odot}$ star is simulated for a period of several hundred milliseconds, starting at a time about 10 milliseconds after core bounce. By that time the prompt shock wave has transformed into a standing accretion shock at a radius of about $115 \mathrm{~km}$, enclosing a mass of $1.25 M_{\odot}$. The matter behind the shock has small negative velocities and settles onto the forming neutron star. The initial model was provided by Bruenn (1993, private communication; see also Bruenn 1992), who computed core collapse and core bounce with use of the equation of state developed by Lattimer \& Swesty (1991). Following the further history for nearly $700 \mathrm{~ms}$ Bruenn did not find an explosion of the star.

As described above, the neutrino luminosities are dependent on convective processes around the neutrino sphere (see Janka 1993, Janka \& Müller 1992) and cannot accurately be determined without an elaborate treatment of neutrino transfer in this turbulent region. Possibly even methods to simulate neutrino transport in more than one dimension have to be applied. Therefore, we decided to vary the neutrino luminosities and to study their influence on the destiny of the shock. For this purpose it is adequate to cut out the inner part of the collapsed stellar core, which also helps to save computer time when performing a large number of one-dimensional runs or multi-dimensional simulations. We put the inner boundary to a density of about $10^{12} \mathrm{~g} / \mathrm{cm}^{3}$, corresponding to an initial radius of $32 \mathrm{~km}$ in the considered model. The radius of the inner boundary was either kept fixed, or was allowed to shrink with time to mimic the contraction of the cooling neutron star. Although we located the inner boundary somewhat inside the neutrino sphere, our computational domain did only partially encompass the convective layer in the protoneutron star. Prescriptions for time-dependent fluxes and spectra of the neutrino emission from the inner core were imposed. While the spectra were not changed in different runs, the neutrino luminosities were varied to study their influence.

The simulations (see Sect. 3) were performed by treating the neutrinos in a 'lightball' approximation: The fluxes entering the computational grid at the inner boundary were kept constant with radius (except for Doppler shift and gravitational red shift effects). Although not being correct, this approach is not too bad outside the neutrino sphere and reduces the computational effort, in particular in multi-dimensional simulations. Since we are not interested in the question, how these fluxes are formed, but in their effect on the shock evolution, the neutrino lightball approximation is very likely justified. The validity of this approximation will be tested in forthcoming simulations (Janka \& Müller, in preparation). All types of neutrinos $\left(\nu_{e}, \bar{\nu}_{e}, \nu_{\mu}, \bar{\nu}_{\mu}, \nu_{\tau}, \bar{\nu}_{\tau}\right)$ were included, and the processes taken into account were electron neutrino/antineutrino absorption on neutrons/protons, neutrino scattering off neutrons, protons, nuclei, electrons, and positrons, and neutrino pair production and pair annihilation by/into electron-positron pairs. Our simulations were performed with an elaborate, vectorized equation of state, which contains contributions by neutrons, protons, alpha particles and a representative heavy nucleus in nuclear statistical equilibrium. Electrons, treated as an arbitrarily degenerate and relativistic gas, and positrons and photons were taken into account.

We employed two different numerical methods for solving the equations of Newtonian hydrodynamics: An explicit, second order Lagrangian scheme with automatic, conservative rezoning, which handles shocks by use of a tensor artificial viscosity (see Janka et 
TABLE 1. Model data for one-dimensional (letter ' $O$ ' in the model name) and two-dimensional (' $T$ ') simulations. The letter ' $f$ ' indicates that the radius of the inner boundary was kept fixed, ' $c$ ' marks model runs with contracting inner boundary. In case of two-dimensional simulations the numbers in parentheses behind the model name give the numbers of angular zones (of one degree size). $L_{\nu_{e}}^{52}$ is the electron neutrino luminosity (in $10^{52} \mathrm{erg} / \mathrm{s}$ ) at the start of the computation, $L_{\nu_{x}}^{52}$ are the individual luminosities of muon and tauon neutrinos and antineutrinos. The luminosity of electron antineutrinos is constrained by the requirement that the inner part of the neutron star with mass $M_{\mathrm{ic}}=0.85 M_{\odot}$ looses an electron fraction of $\Delta Y_{e}$. The total energy loss due to neutrino emission is assumed to equal $\Delta \epsilon \cdot M_{\odot} c^{2} . t_{\exp }$ gives the heating time scale, defined by the moment when the explosion energy $E_{\exp }$ (see text) of the model becomes larger than $10^{48} \mathrm{erg}$. The values in column $E_{\exp }$ give the explosion energies at the end of the computation, i.e. at time $t_{\text {end }}$ after the start of the simulation.

\begin{tabular}{lrrrrrrr}
\hline \hline Model & $\begin{array}{r}L_{\nu e}^{52} \\
{[\mathrm{erg} / \mathrm{s}]}\end{array}$ & $\begin{array}{r}L_{\nu x}^{52} \\
{[\mathrm{erg} / \mathrm{s}]}\end{array}$ & DeltaYe & Deltat & $\begin{array}{r}t_{\text {exp }} \\
{[\mathrm{ms}]}\end{array}$ & $\begin{array}{r}t_{\text {end }} \\
{[\mathrm{ms}]}\end{array}$ & $\begin{array}{r}E_{\text {exp }}\left(t_{\text {end }}\right) \\
{\left[10^{50} \mathrm{erg}\right]}\end{array}$ \\
\hline O1f & 1.850 & 1.650 & 0.05 & 0.035 & $\infty$ & 462. & 0.0 \\
O2f & 1.900 & 1.700 & 0.05 & 0.035 & 270. & 796. & 7.1 \\
O3f & 1.925 & 1.725 & 0.05 & 0.035 & 211. & 735. & 8.2 \\
O4f & 2.750 & 2.250 & 0.25 & 0.150 & 48. & 635. & 48.3 \\
O1c & 1.900 & 1.700 & 0.05 & 0.035 & $\infty$ & 341. & 0.0 \\
O2c & 2.000 & 1.800 & 0.05 & 0.035 & $\infty$ & 365. & 0.0 \\
O3c & 2.100 & 1.850 & 0.06 & 0.045 & $\infty$ & 372. & 0.0 \\
O4c & 2.200 & 1.875 & 0.06 & 0.045 & 162. & 697. & 4.9 \\
O5c & 2.225 & 1.875 & 0.06 & 0.045 & 122. & 927. & 9.5 \\
O6c & 2.250 & 1.900 & 0.07 & 0.050 & 97. & 485. & 13.0 \\
T1f(90) & 1.900 & 1.700 & 0.05 & 0.035 & 93. & 720. & 8.9 \\
T1c(90) & 1.900 & 1.700 & 0.05 & 0.035 & 88. & 214. & 0.9 \\
T2c(180) & 1.900 & 1.700 & 0.05 & 0.035 & 85. & 199. & 1.2 \\
\hline \hline
\end{tabular}

al. 1993). Multi-dimensional simulations were performed with a modified version of the explicit PROMETHEUS code (Fryxell et al. 1989), which is a Eulerian implementation of the Piecewise Parabolic Method by Colella \& Woodward (1984). Comparing results of both codes yields excellent agreement where it required (see also Janka et al. 1993).

\section{Shock Evolution in One- and Two-Dimensional Simulations}

\subsection{Spherically Symmetric Case}

A set of one-dimensional simulations was performed changing the neutrino fluxes from the inner boundary from small values to successively larger ones. The effect on the standing accretion shock was investigated. The results are collected in Table 1 . The model sequence ' $O \# f$ ' was computed with a fixed radius of the inner boundary $R_{\mathrm{ib}}=31.7 \mathrm{~km}$, while the inner boundary was contracting in models ' $\mathrm{O \# c}$ '. The initial electron neutrino luminosity $L_{\nu_{e}}$ and muon and tauon neutrino/antineutrino luminosities $L_{\nu_{x}}$ can also be found in Table 1. The luminosities of all neutrino kinds were assumed to decay exponentially and were constrained by the requirements that a total lepton number $\Delta Y_{e} \cdot \mathcal{A} M_{\mathrm{ic}} c^{2}\left(M_{\mathrm{ic}}=0.85 M_{\odot}, \mathcal{A}\right.$ is Avogadro's number) and a total energy $\Delta \epsilon \cdot M_{\odot} c^{2}$ is radiated away. Numbers for $\Delta Y_{e}$ and $\Delta \epsilon$ are listed in in Table 1.

The models do not explode for low values of the neutrino luminosity. The neutrino emission has to surpass a well defined threshold value to drive a strong expansion of the 


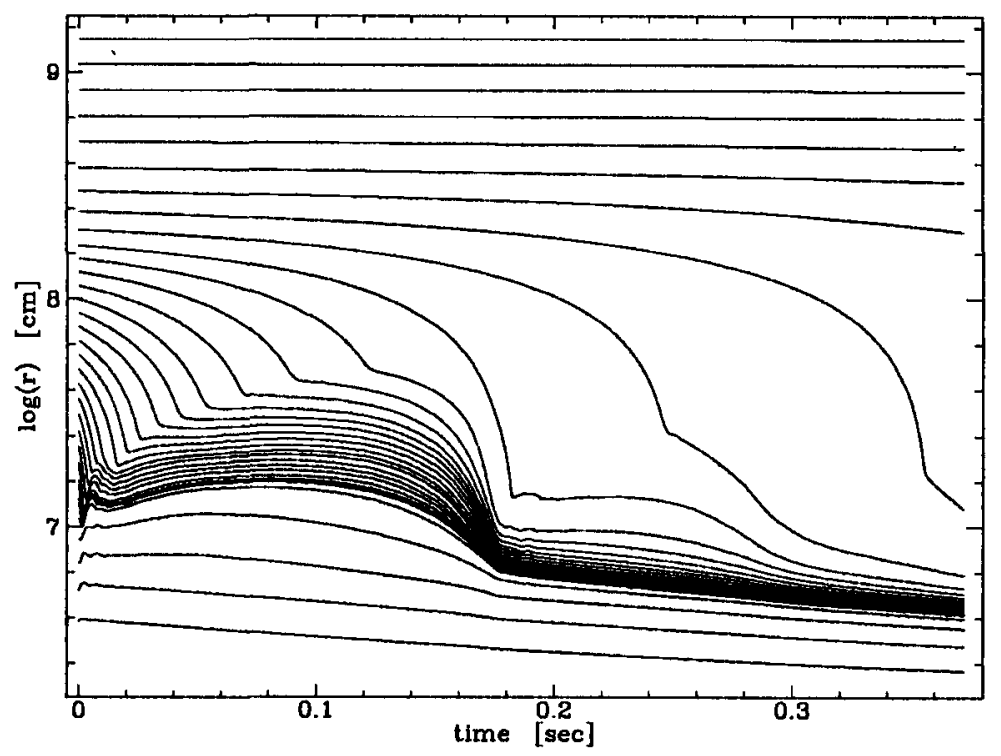

Figure 1. Radial positions as functions of time for a sample of mass shells of model O3c (see Table 1). The protoneutron star is sitting at the bottom of the figure, and the position of the supernova shock can be identified at the sharp turns of the curves. The shock exhibits an oscillatory motion and the model does not explode.

layers between neutrino sphere and standing accretion shock against the ram pressure of the material falling into the shock. Only if this expansion is sufficiently fast and strong, a successful explosion is initiated. Examples for both cases, no explosion and explosion, are shown in Figs. 1 and 2 . In case of failure the region between neutrino sphere and shock expands and contracts, and the shock position exhibits an oscillatory behaviour (see Fig. 1; model O3c in Table 1). Phases of expansion and net energy gain of the matter alternate with compression and accompanying neutrino energy loss. In contrast, when the neutrino heating is powerful enough, the strong expansion prevents the re-emission of energy deposited by neutrino absorption outside the so called 'gain radius'. This location defines the point in the protoneutron star atmosphere, where neutrino cooling - which dominates at high densities and gas temperatures - is exceeded by neutrino heating (see Bethe \& Wilson 1985, Bethe 1990). With the expansion setting in, the temperatures and densities decrease, which maintains favourable conditions for continued neutrino heating. This defines a runaway situation, and finally the shock wave propagates outward to trigger the explosion of the star (Fig. 2; model O4c in Table 1). These results are obtained for the idealized case that the neutrino fluxes originate from an independent central source and are not influenced by the expansion or contraction of the protoneutron star atmosphere. The exact and detailed post bounce history may depend on the coupling between neutrino fluxes and hydrodynamical evolution. Nevertheless, our results show principle features as they were found in the computations by Wilson et al. (1986). Comparison with these, however, reveals some fundamental differences, too. In the simulations by Wilson et al. (1986) much of the neutrino luminosity was produced by spherical accretion of matter falling through the standing accretion shock onto the new-formed neutron star. When neutrino heating began to drive an expansion, this accretion was turned off and the luminosities dropped. Neutrino cooling took over, and the matter started to contract, again increasing the accretion rate. This resulted in an oscillatory up and down of the 


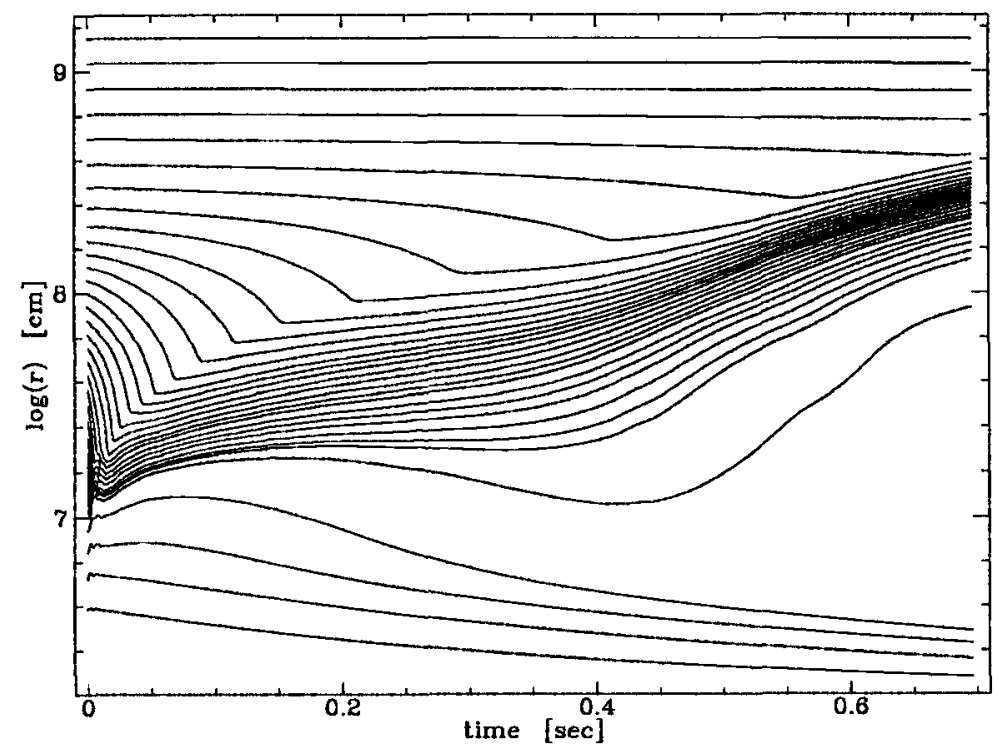

Figure 2. Radial positions $r(t)$ for a sample of mass shells of model O4c (see Table 1). After about $400 \mathrm{~ms}$ post bounce a strong bifurcation between protoneutron star and stellar mantle develops and the model explodes.

shock front in some of the models of Wilson et al. (1986). With our assumption of the neutrino luminosities being produced in the convective mantle region of the protoneutron star we do not have this negative feedback between neutrino emission and matter motion: When a strong neutrino-driven expansion sets in, the matter temperature drops, neutrino re-emission decreases, and neutrino heating by the unchanged neutrino fluxes continues the positive energy transfer to the stellar gas.

This mechanism implies a strong sensitivity to the neutrino luminosities. The explosion energy $E_{\text {exp }}$, which is given by the total energy (kinetic plus internal energies minus gravitational binding energy) of all zones with positive values of this energy, increases steeply for higher neutrino fluxes. In Table 1 the corresponding numbers are listed for the final models (column $t_{\text {end }}$ gives the time at the end of the simulation runs, measured from the beginning of each computation). The time scale $t_{\exp }$ in Table 1 measures the time to start the explosion and is defined as the moment when $E_{\exp }=10^{48} \mathrm{erg}$. This time shrinks for stronger neutrino heating; in any case, however, it was found to be longer than 50 milliseconds. It is remarkable that the threshold value of the neutrino luminosity depends on the treatment of the inner boundary. With fixed radius $R_{\mathrm{ib}}$ our one-dimensional models explode for $L_{\nu_{e}} \gtrsim 1.9 \cdot 10^{52} \mathrm{erg} / \mathrm{s}$, whereas the shrinking inner core prevents explosions below $L_{\nu_{e}} \approx 2.2 \cdot 10^{52} \mathrm{erg} / \mathrm{s}$.

\subsection{Two-Dimensional Case}

In a second set of runs we switched to two dimensions (models ' $T \# \mathbf{f}$ ', ' $T \# c$ ' in Table 1), again treating the two possibilities of fixed inner boundary radius (' $\mathrm{T} \# \mathrm{f}$ ') and contraction of the inner boundary (' $\mathrm{T} \# \mathrm{c}$ '). The numbers in parentheses, which follow the model name, indicate the angular size of the grid (90 or 180 degrees); the angular resolution was one degree. As in one dimension, the model did not explode without neutrino heating in two dimensions. When including neutrinos we chose the electron neutrino luminosity to be $L_{\nu_{e}}=1.9 \cdot 10^{52} \mathrm{erg} / \mathrm{s}$. For this case we found an explosion in the spherically 


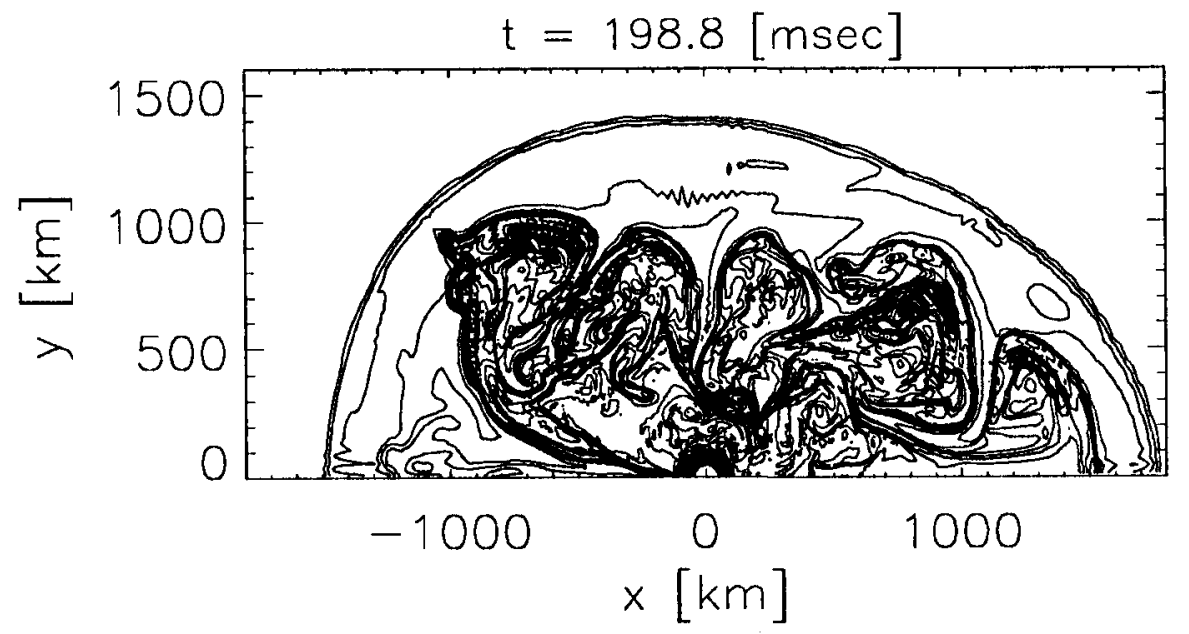

Figure 3. Convective overturn between protoneutron star (in the center) and outgoing supernova shock in model $\mathrm{T} 2 \mathrm{c}(180)$ (see Table 1) at time $t=199 \mathrm{~ms}$ after the start of the computation. The contours correspond to 25 equispaced levels of constant entropy between $s_{\min } \approx 5 k_{\mathrm{B}} /$ nucleon and $s_{\max } \approx 17.3 k_{\mathrm{B}} /$ nucleon. Narrow tubes of down-flowing matter and large rising bubbles occur.

symmetric situation, when the radius of the inner boundary was kept constant (model $\mathrm{O} 2 \mathrm{f}$ ), although it took as long as $t_{\exp }=270 \mathrm{~ms}$ to start the finally successful expansion. The model with the same neutrino parameters, but with contracting inner boundary, model O1c, did not explode. This appeared to us as an interesting case to be studied in two dimensions.

Neutrino heating produces a region with relatively high entropies per particle outside the gain radius; typical values are around $10-15 k_{\mathrm{B}}$ per nucleon, whereas the matter downstream of the standing accretion shock has entropies of about $6-7 k_{\mathrm{B}} /$ nucleon. Such a situation is convectively unstable. In fact, this is confirmed by our two-dimensional simulations, where convective overturn sets in within a few ten milliseconds. In the beginning, the convective mixing encompasses the whole region between gain radius and standing accretion shock. This moves cold (low entropy) material from behind the shock into the heating region and transports hot matter outwards. The convective velocities are several times $10^{8} \mathrm{~cm} / \mathrm{s}$ up to about $10^{9} \mathrm{~cm} / \mathrm{s}$, which is close to the local speed of sound. Without any doubt, this effect aids the explosion; this is clearly visible by comparison of the numbers for $t_{\exp }$ and $E_{\exp }$ for models O2f and T1f: The explosion starts more easily and the explosion energy tends to be higher in the two-dimensional case. Even more dramatic, we found an explosion also in case of a shrinking central core $\left(R_{\mathrm{ib}}\right.$ decreasing with time), i.e. in a situation where the spherically symmetric model O1c failed: Models $\mathrm{T} 1 \mathrm{c}(90)$ and $\mathrm{T} 2 \mathrm{c}(180)$ show a strengthening of the shock wave and an explosive expansion on a time scale close to that of model T1f.

When the shock starts to move out and a dynamical expansion of the post-shock region begins, the expansion time scale becomes shorter than the typical growth time scale of instabilities. This is the moment when the convective pattern is 'frozen in': The number of structures with upward and downward flows as well as their angular sizes and locations on the sphere essentially do not change during the later evolution. Figure 3 shows the region of convective overturn between central neutron star and outgoing shock for model 
$\mathrm{T} 2 \mathrm{c}(180)$ at the end of the computation $t_{\mathrm{end}}=199 \mathrm{~ms}$. We found the structure of the turbulence pattern to be sensitive to the prescribed behaviour of the inner boundary, and dependent on the angular size of the computational grid. Due to the large pressure scale height the structures tend to become quite extended, typical angular diameters being about 30 degrees (compare Fig. 3). With a grid of 90 degrees the structures are artificially confined and cannot fully develop. This reduces the efficiency of the convective transport and explains the difference in the numbers for models $\mathrm{T} 1 \mathrm{c}(90)$ and $\mathrm{T} 2 \mathrm{c}(180)$.

\section{Summary and Conclusions}

We have performed one- and two-dimensional hydrodynamical simulations of the revival phase of the stalled prompt supernova shock in a collapsed $1.31 M_{\odot}$ stellar iron core.

The results for the spherically symmetric situation demonstrate that the possibility of a successful explosion by the neutrino-driven mechanism depends extremely sensitively on the neutrino luminosities during a phase of 100-300 milliseconds after core bounce. If the luminosities fall below a threshold value, the mechanism fails to explode the star. However, if the luminosities lie above this threshold and initiate a sufficiently strong expansion, then favourable conditions for continuing neutrino heating are established and (energetic) explosions result, provided the neutrino luminosities do not drop when the expansion sets in. This is the case when the neutrino flux is mainly originating from a convective region in the protoneutron star, but it is not the case, if the main contribution to the neutrino emission is generated by spherical accretion of infalling matter onto the protoneutron star (as in one-dimensional simulations). Convection in the protoneutron star (Burrows 1987, Burrows \& Fryxell 1992, Janka \& Müller 1992, Janka 1993) transports energy from deeper layers up to the neutrino decoupling region more efficiently than it is done by neutrino diffusion. Therefore convection around and below the neutrino sphere should be the crucial ingredient to neutrino-driven supernova explosions.

Comparing the one-dimensional simulations with the two-dimensional ones we learn that convection outside the protoneutron star clearly and strongly aids the explosion. It transports hot matter from the region of powerful neutrino heating (at the gain radius) into layers farther out behind the shock front. This changes the $\nu$-luminosity threshold for the explosion as well as explosion energies and explosion time scales, and is also crucial to determine the mass of the neutron star remnant. In each of our simulation, oneand two-dimensional, it took at leas. 50 milliseconds for positive explosion energies to develop. This appears to us as the typical neutrino heating time scale required to start the explosion in the considered model. We conclude that high neutrino luminosities from the neutron star have to be maintained for a period which is a multiple of this time scale. Typically, after several hundred milliseconds the expanding material outside the protoneutron star had accumulated an energy comparable with standard type-II supernova energies. For cases of successful explosions the explosion energy is extremely sensitive to the luminosity of the neutrino source. It therefore does not seem likely that neutrino driven type-II supernovae have a uniform value of the explosion energy.

We stress that the effects in two dimensions, quantitatively and qualitatively, depend on the handling of the inner boundary in simulations where the high-density central part of the protoneutron star is cut out. Moreover, they also depend on the angular size of the grid for the simulation. Due to the large pressure scale height in the expanding region between gain radius and shock position the convective overturn proceeds in long tubes of down-flowing material and large rising bubbles. Typical angular diameters of these 
structures were found to be about 30 degrees. On an angular grid of 90 degrees (instead of 180 degrees) the convection could not be adequately simulated, but was less violent and less effective in transporting energy into the shock front. We suspect that opening an additional degree of translational freedom by going to three dimensions could have a similar influence on the convection as extending the angular size of the grid. This may be taken as an indication that a satisfactory numerical treatment might actually require three-dimensional simulations.

\section{Acknowledgements}

H.-Th. J. would like to thank Prof. K. Nomoto and all the organizers of the IAU Colloquium No. 145 for the great opportunity to visit China.

\section{REFERENCES}

Bethe, H. A. 1990, Rev. Mod. Phys., 62, 801

Bethe, H. A., Wilson J.R. 1985, ApJ, 295, 14.

Bruenn, S. W. 1989a, ApJ, 340, 955

Bruenn, S. W. 1989b, ApJ, 341, 385

Bruenn, S. W. 1992, Numerical Simulations of Core Collapse Supernovae, in Proc. of 1st Symposium on Nuclear Physics in the Universe, Oak Ridge, Tennessee, Sept. 1992

Burrows, A. 1987, ApJ, 318, L57

Burrows, A., \& Lattimer, J. M. 1988, Phys. Rep., 163, 51

Burrows, A., \& Fryxell, B. A. 1992, Science, 258, 430

Colella, Ph., \& Woodward, P. R. 1984, J. Comp. Phys., 54, 174

Fryxell, B. A., Müller, E.,\& Arnett, D. 1989, Hydrodynamics and Nuclear Burning, MPApreprint, 449, Max-Planck-Institut für Astrophysik, Garching.

Herant, M., Benz, W., \& Colgate, S. 1992, ApJ, 395, 642.

Janka, H.-Th. 1993, Neutrinos from Type-II Supernovae and the Neutrino-Driven Supernova Mechanism, in Frontier Objects in Astrophysics and Particle Physics, Conf. Proc. Vol. 40, Eds. F. Giovannelli \& G. Mannocchi, SIF, Bologna, p. 345

Janka, H.-Th., \& Müller, E. 1992, Neutrino-Driven Type-II Supernovae Neutrino Heating and Post Bounce Dynamics, in Frontiers of Neutrino Astrophysics, Univ. Academy Press, Tokyo, Japan (in press); MPA-preprint, 711

Janka, H.-Th., Zwerger, Th., \& Mönchmeyer, R. 1993, A\&A, 268, 360

Lattimer, J. M., \& Swesty, F. D. 1991, Nucl. Phys., A535, 331

Mayle, R. W. 1985, Ph.D. Thesis, UC Berkeley, UCRL report no. 53713

Müller, E. 1993, Two- and Three-Dimensional Simulations of Convection in Protoneutron Stars, in Proc. of 7th Workshop on Nuclear Astrophysics, Ringberg Castle, Tegernsee, March 1993, MPA-report, in press

Wilson, J. R., Mayle, R. W., Woosley, S. E., Weaver, T. 1986, Ann. N.Y. Acad. Sci., 470, 267 